\title{
A STABILITY THEOREM OF THE NAVIER-STOKES FLOW PAST A ROTATING BODY
}

\author{
YOSHIHIRO SHIBATA \\ Department of Mathematics, Waseda University \\ Shinjuku-ku, Tokyo, 169-8555, Japan \\ E-mail: yshibata@waseda.jp
}

\begin{abstract}
In this paper, a stability theorem of the Navier-Stokes flow past a rotating body is reported. Concerning the linearized problem, the proofs of the generation of a $C_{0}$ semigroup and its decay properties are sketched.
\end{abstract}

1. Introduction. Consider a rigid body $\mathcal{R}$ moving through an incompressible viscous fluid $\mathcal{L}$ that fills the whole three-dimensional space exterior to $\mathcal{R}$. We assume that with respect to a frame attached to $\mathcal{R}$, the translational velocity $u_{\infty}$ and the angular velocity $\omega$ of $\mathcal{R}$ are both constant vectors. Without loss of generality we may assume that $\omega=$ ${ }^{T}(0,0, a),{ }^{T} M$ denoting the transposed $M$. If the flow is non-slip at the boundary, then the motion is described by the following equation:

$$
\begin{aligned}
& v_{t}+v \cdot \nabla v-\Delta v+\nabla \pi=g \quad \text { in } \quad \Omega(t), \quad t>0, \\
& \operatorname{div} v=0 \quad \text { in } \quad \Omega(t), \quad t>0, \\
& v(y, t)=\omega \times y \quad \text { on } \quad \partial \Omega(t), \quad t>0, \\
& v(y, t) \rightarrow u_{\infty} \neq 0 \quad \text { as } \quad|y| \rightarrow \infty, \quad t>0, \\
& v(y, 0)=v_{0}(y) \quad \text { on } \Omega \text {, }
\end{aligned}
$$

in the time-dependent exterior domain

$$
\Omega(t)=\mathcal{O}(a t) \Omega
$$

2000 Mathematics Subject Classification: Primary 35Q30; Secondary 76D05, 76D07.

Key words and phrases: $C^{0}$ semigroup, $L_{p}-L_{q}$ estimate, rotating body, exterior domain, Oseen equations.

Partly supported by Grant-in-Aid for Scientific Research (B) - 15340204, Japan Society for the Promotion of Science.

The paper is in final form and no version of it will be published elsewhere. 
where $\mathcal{O}(t)$ denotes the orthogonal matrix

$$
\mathcal{O}(t)=\left(\begin{array}{ccc}
\cos t & -\sin t & 0 \\
\sin t & \cos t & 0 \\
0 & 0 & 1
\end{array}\right)
$$

and $\Omega$ is a fixed exterior domain in $\mathbf{R}^{3}$ with $C^{1,1}$ boundary $\partial \Omega$. Over the last several years, the study of well-posedness of the initial boundary value problem (1) and related topics has attracted the attention of several authors; see, e.g. [1]-[37]. Besides the intrinsic mathematical interest, this is probably also due to the fact that problem (1) is at the foundation of several important engineering applications. The interested reader is referred to [11] and to the literature cited therein.

To treat (1) in the time-independent domain $\Omega$, we introduce

$$
\begin{aligned}
x & =\mathcal{O}(t)^{T} y, \\
u(x, t) & =\mathcal{O}(t)^{T}\left(v(y, t)-u_{\infty}\right), \\
p(x, t) & =\pi(y, t) .
\end{aligned}
$$

Then, we see that $(u, p)$ satisfies the modified Navier-Stokes equations:

$$
\begin{array}{rlrl}
u_{t}+u \cdot \nabla u-\Delta u+\left(\mathcal{O}(t)^{T} u_{\infty}\right) \cdot \nabla u & & \\
-(\omega \times x) \cdot \nabla u+\omega \times u+\nabla \pi & =f & & \text { in } \Omega \times(0, \infty), \\
\operatorname{div} u & =0 & & \text { in } \Omega \times(0, \infty), \\
u(x, t) & =\omega \times x-\mathcal{O}(t) u_{\infty} & & \text { on } \partial \Omega \times(0, \infty), \\
u(x, t) & \rightarrow 0 & & \text { as }|x| \rightarrow \infty, t>0, \\
u(x, 0) & =v_{0}(x) & & \text { in } \Omega .
\end{array}
$$

In this paper, we consider only the case where

$$
u_{\infty}=k \mathbf{e}_{3}
$$

with $\mathbf{e}_{3}={ }^{T}(0,0,1)$ so that

$$
\mathcal{O}(t)^{T} u_{\infty}=k \mathbf{e}_{3} \quad \text { for all } t>0 .
$$

Therefore, the equation (1) leads to the system:

$$
\begin{array}{rlrl}
u_{t}+u \cdot \nabla u-\Delta u+k \partial_{3} u & & \\
-(\omega \times x) \cdot \nabla u+\omega \times u+\nabla \pi & =f & & \\
\operatorname{div} u & =0 & & \text { in } \Omega \times(0, \infty), \\
u(x, t) & =\omega \times x-k \mathbf{e}_{3} & & \text { on } \partial \Omega \times(0, \infty), \\
u(x, t) & \rightarrow 0 & & \text { in } \Omega \times(0, \infty), \\
u(x, 0) & =v_{0}(x) & & \text { in } \Omega .
\end{array}
$$

First of all, we would like to recall some stability theorem for (4) in the case where $\omega=0$, that is, we consider the Navier-Stokes flow just past a rigid body without rotation. In this case, our equation becomes the following one:

$$
\begin{aligned}
u_{t}+u \cdot \nabla u-\Delta u+k \partial_{3} u & =f & & \text { in } \Omega \times(0, \infty), \\
\operatorname{div} u & =0 & & \text { in } \Omega \times(0, \infty), \\
u(x, t) & =-k \mathbf{e}_{3} & & \text { on } \partial \Omega \times(0, \infty), \\
u(x, t) & \rightarrow 0 & & \text { as }|x| \rightarrow \infty, t>0, \\
u(x, 0) & =v_{0}(x) & & \text { in } \Omega .
\end{aligned}
$$


In order to solve the problem (5) with $f=f(x)$, we have to solve the stationary problem:

$$
\begin{array}{rlrl}
v(x) \cdot \nabla v(x)-\Delta v(x)+k \partial_{3} v(x)+\nabla p & =f(x), & & \operatorname{div} v(x)=0 \\
v(x) & =-k \mathbf{e}_{3} & & \text { in } \Omega, \\
v(x) & \rightarrow 0 & & \text { on } \partial \Omega, \\
v(x) & & \text { as }|x| \rightarrow \infty .
\end{array}
$$

Shibata [48] proved the following two theorems.

TheOREM 1. Let $3<q<\infty$ and let $\delta$ and $\beta$ be any numbers such that $0<\delta<1 / 4$ and $0<\delta<\beta<1-\delta$. Let $f \in L_{\infty}(\Omega)^{3}$. Then, there exists a constant $\epsilon, 0<\epsilon \leq 1$, depending on $q, \delta$ and $\beta$ but independent of $k$ such that if $0<k \leq \epsilon$ and $\langle\langle f\rangle\rangle_{2 \delta} \leq \epsilon|k|^{\beta+\delta}$, then the problem (6) admits a unique solution $(v, p)$ possessing the estimate:

$$
\|v\|_{W_{q}^{2}(\Omega)}+\|\| v\|\|_{\delta}+\|\nabla p\|_{L_{q}(\Omega)} \leq|k|^{\beta}
$$

where

$$
\begin{aligned}
\langle\langle f\rangle\rangle_{2 \delta}= & \sup _{x \in \Omega}(1+|x|)^{\frac{5}{2}}\left(1+\left(|x|-x_{3}\right)\right)^{\frac{1}{2}+2 \delta}|f(x)|, \\
\||| v \mid\|_{\delta}= & \sup _{x \in \Omega}(1+|x|)\left(1+\left(|x|-x_{3}\right)\right)^{\delta}|v(x)| \\
& +\sup _{x \in \Omega}(1+|x|)^{\frac{3}{2}}\left(1+\left(|x|-x_{3}\right)\right)^{\frac{1}{2}+\delta}|\nabla v(x)| .
\end{aligned}
$$

To solve (5), we set $u(x, t)=v(x)+w(x, t)$ and $\pi(x, t)=p(x)+\theta(x, t)$, and then $(w, \theta)$ should satisfy the equations:

$$
\begin{aligned}
w_{t}+w \cdot \nabla w-\Delta w+k \partial_{3} w & & & \\
+v \cdot \nabla w+w \cdot \nabla v+\nabla \theta & =0 & & \text { in } \Omega \times(0, \infty), \\
\operatorname{div} w & =0 & & \text { in } \Omega \times(0, \infty), \\
w & =0 & & \text { on } \partial \Omega \times(0, \infty), \\
w(x, t) & \rightarrow 0 & & \text { as }|x| \rightarrow \infty, t>0, \\
w(x, 0) & =w_{0}(x)=v_{0}(x)-v(x) & & \text { in } \Omega .
\end{aligned}
$$

The corresponding linearized equation for (7) is the usual Oseen equation:

$$
\begin{aligned}
w_{t}-\Delta w+k \partial_{3} w+\nabla \theta & =0 & & \text { in } \Omega \times(0, \infty), \\
\operatorname{div} w & =0 & & \text { in } \Omega \times(0, \infty), \\
w & =0 & & \text { on } \partial \Omega \times(0, \infty), \\
w(x, t) & \rightarrow 0 & & \text { as }|x| \rightarrow \infty, t>0, \\
w(x, 0) & =a(x) & & \text { in } \Omega .
\end{aligned}
$$

Let $P$ be the Helmholtz projection from $L_{q}(\Omega)^{3}$ onto the solenoidal space $J_{q}(\Omega)(1<q<$ $\infty)$, where

$$
\begin{aligned}
J_{q}(\Omega) & =\text { the completion of } C_{0, \sigma}^{\infty}(\Omega) \text { in } L_{q}(\Omega)^{3}, \\
C_{0, \sigma}^{\infty}(\Omega) & =\left\{u \in C_{0}^{\infty}(\Omega)^{3} \mid \operatorname{div} u=0 \text { in } \Omega\right\}
\end{aligned}
$$

(cf. [40], [42], [47], [50]). Let us define the Oseen operator by means of the formula:

$$
\begin{aligned}
O_{k} u & =P\left(-\Delta u+k \partial_{3} u\right) \quad \text { for } u \in \mathcal{D}\left(O_{k}\right), \\
\mathcal{D}\left(O_{k}\right) & =W_{q}^{2}(\Omega)^{3} \cap W_{q, 0}^{1}(\Omega)^{3} \cap J_{q}(\Omega),
\end{aligned}
$$


where $W_{q, 0}^{1}(\Omega)^{3}=\left\{u \in W_{q}^{1}(\Omega)^{3}|u|_{\partial \Omega}=0\right\}$. Then, the equation (8) can be written as the evolution equation:

$$
w_{t}(t)+O_{k} w(t)=0 \quad \text { for } t>0, \quad w(0)=a, \quad w(t) \in \mathcal{D}\left(O_{k}\right)(t>0) .
$$

We know that $O_{k}$ generates an analytic semigroup $\left\{T_{k}(t)\right\}_{t \geq 0}$ on $J_{q}(\Omega)$. Moreover, we know the following theorem which was proved by Kobayashi and Shibata [45].

THEOREM 2. Let $k_{0}$ be any positive number and assume that $0 \leq k \leq k_{0}$. Then, the following estimates hold for $f \in J_{q}(\Omega)$ and $t>0$ :

$$
\begin{aligned}
\left\|T_{k}(t) f\right\|_{L_{r}(\Omega)} & \leq C_{q, r} t^{-\frac{3}{2}\left(\frac{1}{q}-\frac{1}{r}\right)}\|f\|_{L_{q}(\Omega)} \quad(1<q \leq r \leq \infty, q \neq \infty), \\
\left\|\nabla T_{k}(t) f\right\|_{L_{r}(\Omega)} & \leq C_{q, r} t^{-\frac{1}{2}-\frac{3}{2}\left(\frac{1}{q}-\frac{1}{r}\right)}\|f\|_{L_{q}(\Omega)} \quad(1<q \leq r \leq 3) .
\end{aligned}
$$

Here, the constant $C_{q, r}$ depends on $k_{0}$ whenever $|k| \leq k_{0}$ but is independent of $k, t$ and $f$.

Applying Duhamel's principle to (7), we have the integral equation:

$$
w(t)=T_{k}(t) w_{0}-\int_{0}^{t} T_{k}(t-s) P[L[v] w(s)+w(s) \cdot \nabla w(s)] d s
$$

where we have set

$$
L[v] w(s)=v \cdot \nabla w(s)+w(s) \cdot \nabla v .
$$

Applying the Kato argument in [44] to (11) and using Theorem 1 and Theorem 2, we have the following theorem which was proved by Shibata [48, Theorem 1.4].

Theorem 3. Let $3<q<\infty$ and let $\delta$ and $\beta$ be the same as in Theorem 1. In addition, we assume that $0<\delta<\min (1 / 6,4 / q)$. Let $f \in L_{\infty}(\Omega)^{3}$ and $w_{0} \in J_{3}(\Omega)$. Then, there exists an $\epsilon>0,0<\epsilon \leq 1$, depending only on $q, \beta$ and $\delta$ essentially such that if $0<k \leq \epsilon,\langle\langle f\rangle\rangle_{2 \delta} \leq \epsilon k^{\beta+\delta}$ and $\left\|w_{0}\right\|_{3} \leq \epsilon$, then the problem (11) admits a unique solution $w \in B C\left([0, \infty), J_{3}(\Omega)\right)$ possessing the following properties:

$$
\begin{aligned}
& {[w]_{3,0, t}+[w]_{r, \mu(r), t}+[\nabla w]_{3,1 / 2, t} \leq \sqrt{\epsilon} \quad \text { for any } t>0,} \\
& \lim _{t \rightarrow 0+}\left(\left\|w(\cdot, t)-w_{0}\right\|_{L_{3}(\Omega)}+[w]_{r, \mu(r), t}+[\nabla w]_{3,1 / 2, t}\right)=0 .
\end{aligned}
$$

Here we have set

$$
\begin{gathered}
{[z]_{r, \rho, t}=\sup _{0<s<t} s^{\rho}\|z(\cdot, s)\|_{L_{r}(\Omega)},} \\
\mu(r)=\frac{3}{2}\left(\frac{1}{3}-\frac{1}{r}\right)=\frac{1}{2}-\frac{3}{2 r} \quad \text { for } r \geq 3 .
\end{gathered}
$$

2. Main results. Our goal is to extend Theorems 1 and 3 of section 1 to the case where $\omega \neq 0$. But, so far we do not know the existence of solutions to (6) which possess nice decay properties at far field like $v(x)$ stated in Theorem 1 in section 1 . We shall only report the existence of a semigroup and its decay properties like Theorem 2 in section 1. 
As a linearized problem of (5), we consider the linear equations:

$$
\begin{aligned}
& u_{t}+L_{k, a} u+\nabla \pi=0 \text { in } \Omega, t>0 \text {, } \\
& \nabla \cdot u=0 \text { in } \Omega, t>0 \text {, } \\
& u=0 \text { on } \Gamma \text {, } \\
& \left.u\right|_{t=0}=f \text { in } \Omega \text {. }
\end{aligned}
$$

Here,

$$
L_{k, a} u=-\Delta u+k \partial_{3} u-(\omega \times x) \cdot \nabla u+\omega \times u,
$$

$\omega=(0,0, a), k$ and $a$ are real constants, and $u=\left(u_{1}, u_{2}, u_{3}\right)$ and $\pi$ are unknown velocity field and pressure, respectively. The equation in (12) is written componentwise as follows:

$$
\begin{aligned}
& \frac{\partial u_{1}}{\partial t}-\Delta u_{1}+k \frac{\partial u_{1}}{\partial x_{3}}-a\left(x_{1} \frac{\partial u_{1}}{\partial x_{2}}-x_{2} \frac{\partial u_{1}}{\partial x_{1}}\right)-a u_{2}+\frac{\partial \pi}{\partial x_{1}}=0, \\
& \frac{\partial u_{2}}{\partial t}-\Delta u_{2}+k \frac{\partial u_{2}}{\partial x_{3}}-a\left(x_{1} \frac{\partial u_{2}}{\partial x_{2}}-x_{2} \frac{\partial u_{2}}{\partial x_{1}}\right)+a u_{1}+\frac{\partial \pi}{\partial x_{2}}=0, \\
& \frac{\partial u_{3}}{\partial t}-\Delta u_{3}+k \frac{\partial u_{3}}{\partial x_{3}}-a\left(x_{1} \frac{\partial u_{3}}{\partial x_{2}}-x_{2} \frac{\partial u_{3}}{\partial x_{1}}\right)+\frac{\partial \pi}{\partial x_{3}}=0 .
\end{aligned}
$$

To show the existence of solutions to (12), we use the semigroup approach. Since the pressure term $\pi$ has no time evolution, we have to eliminate $\pi$ by using the Helmholtz decomposition. Let $P$ be the Helmholtz projection given in section 1 . Let us define the operator $\mathcal{L}_{k, a}$ by the formula:

$$
\mathcal{L}_{k, a} u=P L_{k, a} u=P\left(-\Delta u+k \partial_{3} u-(\omega \times x) \cdot \nabla u+\omega \times u\right)
$$

with domain:

$$
C D_{q}(\Omega)=\left\{u \in J_{q}(\Omega) \cap W_{q}^{2}(\Omega)|u|_{\partial \Omega}=0, \quad(\omega \times x) \cdot \nabla u \in L_{q}(\Omega)\right\} .
$$

And then, problem (12) is written as follows:

$$
\begin{aligned}
u_{t}+\mathcal{L}_{\Omega} u & =0 \quad \text { in } J_{q}(\Omega) & & \text { for } t>0, \\
\left.u\right|_{t=0} & =f, & & \\
u(t) & \in \mathcal{D}_{q}(\Omega) & & \text { for } t>0 .
\end{aligned}
$$

One of the significant characteristics of the operator $\mathcal{L}_{k, a}$ is that the crucial drift operator $(\omega \times x) \cdot \nabla$ is never subordinate to the viscous term $\Delta$ and equation (13) has both parabolic and hyperbolic features. In fact, the following theorem was proved by Farwig, Nečasová and Neustupa [9], [10].

TheOREM 4. The essential spectrum of $\mathcal{L}_{k, a}$ coincides with

$$
\bigcup_{j=-\infty}^{\infty}\left\{\sqrt{-1} a j+\left\{\lambda \in \mathbf{C} \mid k^{2} \operatorname{Re} \lambda+(\operatorname{Im} \lambda)^{2}>0\right\}\right\} .
$$

From Theorem 4, we know that the operator $\mathcal{L}_{k, a}$ does not generate an analytic semigroup. But, we can show the generation of a continuous semigroup and its decay estimate. Namely, the following two theorems are the main results which the author would like to report in this paper. 
Theorem 5. Let $1<q<\infty$. Then, $\mathcal{L}_{k, a}$ generates a $C^{0} \operatorname{semigroup}\left\{T_{k, a}(t)\right\}_{t \geq 0}$ on $J_{q}(\Omega)$.

THEOREM 6. The following estimates hold for any $f \in J_{q}(\Omega)$ and $t>0$ :

$$
\begin{aligned}
\left\|T_{k, a}(t) f\right\|_{L_{r}(\Omega)} & \leq C_{q, r} t^{-\frac{3}{2}\left(\frac{1}{q}-\frac{1}{r}\right)}\|f\|_{L_{q}(\Omega)} & & (1<q \leq r \leq \infty, q \neq \infty), \\
\left\|\nabla T_{k, a}(t) f\right\|_{L_{r}(\Omega)} & \leq C_{q, r} t^{-\frac{1}{2}-\frac{3}{2}\left(\frac{1}{q}-\frac{1}{r}\right)}\|f\|_{L_{q}(\Omega)} & & (1<q \leq r \leq 3) .
\end{aligned}
$$

Here, the constant $C_{q, r}$ depends on $a_{0}$ and $k_{0}$ whenever $|a| \leq a_{0}$ and $0<k \leq k_{0}$ but is independent of $a, k, t$ and $f$.

REMARK 1. Theorem 5 was first proved by Hishida [19] when $q=2$ and $k=0$. Later on, Geissert-Heck-Hieber [16] proved Theorem 5 when $1<q<\infty$ and $k=0$. Our proof is different from Hishida [19] and Geissert-Heck-Hieber [16] and based on some new considerations of the pressure terms.

The estimates (14) was proved by Iwashita [43] when $a=k=0$, by Kobayashi and Shibata [45] when $a=0$ and $k \neq 0$ and by Hishida and Shibata [24] when $a \neq 0$ and $k=0$. The restriction: $1<q \leq r \leq 3$ of the second inequality in (14) is unavoidable at least in the case where $a=k=0$, which was proved by Maremonti-Solonnikov [46].

3. Remark on a stability theorem. Consider the original non-linear problem (4) in the case where $f=f(x)$. The corresponding stationary problem is given as

$$
\begin{aligned}
w \cdot \nabla w-\Delta w+k \partial_{3} w & & & \\
-(\omega \times x) \cdot \nabla w+\omega \times w+\nabla \theta & =f & & \text { in } \Omega, \\
\operatorname{div} w & =0 & & \text { in } \Omega, \\
w & =\omega \times x-k \mathbf{e}_{3} & & \text { on } \partial \Omega, \\
w(x) & \rightarrow 0 & & \text { as }|x| \rightarrow \infty .
\end{aligned}
$$

Setting $u(x, t)=w(x)+z(x, t)$ and $\pi(x, t)=\theta(x)+\kappa(x, t)$ in (4), we have the equation for $z$ and $\kappa$ as follows:

$$
\begin{array}{rlrl}
z_{t}+z \cdot \nabla z+z \cdot \nabla w+w \cdot \nabla z-\Delta z & & \\
+k \partial_{3} z-(\omega \times x) \cdot \nabla z+\omega \times z+\nabla \kappa & =0 & & \\
\operatorname{div} z & =0 & & \text { in } \Omega \times(0, \infty) \\
z & =\omega \times x-k \mathbf{e}_{3} & & \text { on } \partial \Omega \times(0, \infty), \\
z(t, x) & \rightarrow 0 & & \text { as }|x| \rightarrow \infty, t>0, \\
z(0, x) & =v_{0}(x)-w(x) . & &
\end{array}
$$

Following Kato [44], instead of (16) we consider the integral equation:

$$
z(t)=T_{k, a}(t)\left(v_{0}-w\right)-\int_{0}^{t} T_{k, a}(t-s) P[z(s) \cdot \nabla z(s)+z(s) \cdot \nabla w+w \cdot \nabla z(s)] d s .
$$

Then, using Theorem 6 and employing the same argument as in Shibata [48] we have the following theorem which is an extension of Theorem 3 in section 1 to the case where $\omega \neq 0$.

Theorem 7 (Stability Theorem). Assume that problem (15) admits solutions $w(x)$ and $\theta(x)$. Let $\sigma$ be a small positive number and $3<q<\infty$. Then, there exists a small positive 
number $\epsilon$ depending on $\sigma$ and $q$ such that if $v_{0}-w \in J_{3}(\Omega)$ and

$$
\|w\|_{L_{3-\sigma}(\Omega) \cap L_{3+\sigma}(\Omega)}+\|\nabla w\|_{L_{(3 / 2)-\sigma}(\Omega) \cap L_{(3 / 2)+\sigma^{(}(\Omega)}}+\left\|v_{0}-w\right\|_{L_{3}(\Omega)} \leq \epsilon
$$

then problem (17) admits a unique solution

$$
z(t) \in C\left([0, \infty), J_{3}(\Omega)\right) \cap C\left((0, \infty), L_{q}(\Omega) \cap W_{3}^{1}(\Omega)\right)
$$

such that

$$
\begin{aligned}
{[z]_{3,0, t}+[z]_{q, \mu(q), t}+[\nabla z]_{3,1 / 2, t} } & \leq \sqrt{\epsilon} \quad \text { for any } t>0, \\
\lim _{t \rightarrow 0+}\left\|z(t)-\left(v_{0}-w\right)\right\|_{L_{3}(\Omega)} & =0 \\
\lim _{t \rightarrow 0+}\left([z]_{q, \mu(q), t}+[\nabla z]_{3,1 / 2, t}\right) & =0 .
\end{aligned}
$$

REMARK 2. Galdi and Silvestre [14] proved the existence of solutions to (15), but the velocity fields in [14] behave $O\left(|x|^{-1}\right)$ when $|x| \rightarrow \infty$ and they did not show the asymptotic behaviour of the gradient of the velocity fields. We do not know any existence theorem for solutions to (15) which satisfy (18). But, the author believes that stationary solutions satisfying (18) exist, because the motion is stabilized by translation.

4. An idea of a proof of Theorem 5. In this section, we consider the solvability of the equations:

$$
\begin{aligned}
& u_{t}+L_{k, a} u+\nabla \pi=0, \quad \nabla \cdot u=0 \quad \text { in } \Omega \times \mathbf{R}_{+}, \\
& \left.u\right|_{\partial \Omega}=0,\left.\quad u\right|_{t=0}=f,
\end{aligned}
$$

where $\mathbf{R}_{+}=(0, \infty)$. The main step in our proof of unique existence of solutions to (19) is to show the following theorem.

Theorem 8. Let $1<q<\infty$ and set

$$
L_{q, R-1}(\Omega)=\left\{f \in L_{q}(\Omega)^{3} \mid f(x)=0(|x|>R-1)\right\} .
$$

If $f \in L_{q, R-1}(\Omega) \cap \mathcal{D}_{q}(\Omega)$, then problem (19) admits a unique solution

$$
u \in C^{0}\left([0, \infty), W_{q}^{2}(\Omega)\right) \cap C^{1}([0, \infty)
$$

with some pressure term $\pi$ such that for any $\gamma>0$ the estimate

$$
\|u(t)\|_{W_{q}^{2}(\Omega)}+\left\|u_{t}(t)\right\|_{L_{q}(\Omega)} \leq C_{\gamma} e^{\gamma t}\|f\|_{W_{q}^{2}(\Omega)}
$$

holds with some constant $C_{\gamma}$ depending on $\gamma$.

In order to show Theorem 8, the key step is to show the following theorem.

TheOREM 9. Let $1<q<\infty$. Then, for every $f \in L_{q, R-1}(\Omega)$, problem (19) replacing $f$ by $P f$ admits a unique solution $(u, \pi)$ having the following regularity properties:

$$
\begin{aligned}
& u \in C^{0}\left([0, \infty), J_{q}(\Omega)\right) \cap C^{1}\left(\mathbf{R}_{+}, L_{q}(\Omega)\right) \cap C^{0}\left(\mathbf{R}_{+}, W_{q}^{2}(\Omega)\right), \\
& \pi \in C^{0}\left(\mathbf{R}_{+}, \hat{W}_{q}^{1}(\Omega)\right),
\end{aligned}
$$

and satisfying the following estimates:

$$
\begin{aligned}
& \|u(t)\|_{L_{q}(\Omega)}+t^{1 / 2}\|\nabla u(t)\|_{L_{q}(\Omega)}+t\left(\left\|u_{t}(t)\right\|_{L_{q}(\Omega)}+\|u(t)\|_{W_{q}^{2}(\Omega)}\right. \\
& \left.\quad+\|\nabla \pi(t)\|_{L_{q}(\Omega)}\right) \\
& \leq C_{\gamma} e^{\gamma t\|f\|_{L_{q}(\Omega)}}, \\
& t^{(1 / 2)(1+(1 / q))}\left(\left\|u_{t}(t)\right\|_{L_{q}\left(\Omega_{b}\right)}+\|\pi(t)\|_{L_{q}\left(\Omega_{b}\right)}\right) \leq C_{\gamma, b} e^{\gamma t}\|f\|_{L_{q}(\Omega)}
\end{aligned}
$$


for any $t>0$. Here, $\Omega_{b}=B_{b} \cap \Omega(b>R), \gamma>0$ is any real number, and $C_{\gamma}$ and $C_{\gamma, b}$ are constants depending on not only $\gamma, R, q, b$ but also $a_{0}$ and $k_{0}$ whenever $|a| \leq a_{0}$ and $|k| \leq k_{0}$ but are independent of $a, k, t$ and $f$. Here, we have set

$$
\hat{W}_{q}^{1}(\Omega)=\left\{p \in L_{q, \text { loc }}(\bar{\Omega}) \mid \nabla p \in L_{q}(\Omega)^{3}\right\} .
$$

Proof of Theorem 8. Assuming that Theorem 9 holds, we shall show Theorem 8. Let $f \in L_{q, R-1}(\Omega) \cap \mathcal{D}_{q}(\Omega)$ and let $(u, p)$ be a solution to the equation (19). By Theorem 9, we know the existence of $(u, \pi)$ satisfying $(20)$ and $(21)$. Let $(v, p)$ be a solution to the equation:

$$
\begin{aligned}
& v_{t}+L_{k, a} v+\nabla p=0, \quad \nabla \cdot v=0 \quad \text { in } \Omega \times \mathbf{R}_{+}, \\
& \left.v\right|_{\partial \Omega}=0,\left.\quad v\right|_{t=0}=-P L_{k, a} f .
\end{aligned}
$$

By Theorem 9 we know the existence of $(v, p)$ which also satisfy $(20)$ and (21), replacing $f$ by $P L_{k, a} f$. In particular, we have

$$
\|v(t)\|_{L_{q}(\Omega)} \leq C_{\gamma} e^{\gamma t}\|f\|_{W_{q}^{2}(\Omega)}
$$

because the inequality $\|(\omega \times x) \cdot \nabla f\|_{L_{q}(\Omega)} \leq C\|f\|_{W_{q}^{2}(\Omega)}$ follows from the assumption that $f(x)=0$ for $|x| \geq R-1$. Our task is to show that $u_{t}=v$. To this end, we set

$$
w(x, t)=f(x)+\int_{0}^{t} v(x, s) d z
$$

and we shall show that $w(x, t)=u(x, t)$ in what follows. Given $\varphi \in \mathcal{D}_{q^{\prime}}(\Omega)$, by $(24)$ we have

$$
\frac{d}{d t}(w(t), \varphi)=\left(w_{t}(t), \varphi\right)=(v(t), \varphi)
$$

Here, we have set

$$
(u, v)=\int_{\Omega} u(x) \cdot v(x) d x .
$$

On the other hand, for $t>0$ by (22) we have

$$
\begin{aligned}
\frac{d}{d t}(v(t), \varphi) & =-\left(L_{k, a} v(t)+\nabla p(t), \varphi\right) \\
& =-\left(v(t), L_{k, a}^{*} \varphi\right)+(p, \nabla \cdot \varphi)=-\left(v(t), L_{k, a}^{*} \varphi\right)
\end{aligned}
$$

where we have set $L_{k, a}^{*} \varphi=-\Delta \varphi-k \partial_{3} \varphi+(\omega \times x) \cdot \nabla \varphi-\omega \times \varphi$. Therefore, integrating the above formula with respect to $t$, we have

$$
(v(t), \varphi)=(v(0), \varphi)-\int_{0}^{t}\left(v(s), L_{k, a}^{*} \varphi\right) d s=-\left(P L_{k, a} f, \varphi\right)-\int_{0}^{t}\left(v(s), L_{k, a}^{*} \varphi\right) d s,
$$

which combined with (24) and (25) implies that

$$
\begin{aligned}
\left(w_{t}(t), \varphi\right) & =-\left(P L_{k, a} f, \varphi\right)-\int_{0}^{t}\left(v(s), L_{k, a}^{*} \varphi\right) d s \\
& =-\left(P L_{k, a} f, \varphi\right)+\left(f-w(t), L_{k, a}^{*} \varphi\right) .
\end{aligned}
$$

Since $f \in \mathcal{D}_{q}(\Omega)$ and $\varphi \in \mathcal{D}_{q^{\prime}}(\Omega)$, we have $\left(P L_{k, a} f, \varphi\right)=\left(f, L_{k, a}^{*} \varphi\right)$, and therefore by (27) we have

$$
\frac{d}{d t}(w(t), \varphi)+\left(w(t), L_{k, a}^{*} \varphi\right)=0
$$


Since

$$
\left(u_{t}(t), \varphi\right)+\left(u(t), L_{k, a}^{*} \varphi\right)=0
$$

as follows from $(19),(20),(21)$ and the fact that $\nabla \cdot \varphi=0$, by (28) we have

$$
\left(w_{t}(t)-u_{t}(t), \varphi\right)+\left(w(t)-u(t), L_{k, a}^{*} \varphi\right)=0
$$

for any $\varphi \in \mathcal{D}_{q^{\prime}}(\Omega)$. Now, given any $\psi \in C_{0, \sigma}^{\infty}(\Omega)$ and $T>0$, let $(z, \theta)$ be the solution to the dual problem:

$$
\begin{aligned}
& -z_{t}+L_{k, a}^{*} z+\nabla \theta=0, \quad \nabla \cdot z=0 \quad \text { in } \Omega \text { for } t<T, \\
& \left.z\right|_{\partial \Omega}=0,\left.\quad u\right|_{t=T}=\psi .
\end{aligned}
$$

Then, by (29) and (30) we have

$$
\begin{aligned}
0 & =\int_{0}^{T}\left(w_{t}(t)-u_{t}(t), z(t)\right)+\left(w(t)-u(t), L_{k, a}^{*} z(t)\right) d t \\
& =(w(T)-u(T), z(T))-(w(0)-u(0), z(0))+\int_{0}^{T}\left(w(t)-u(t),-z_{t}(t)+L_{k, a}^{*} z(t)\right) d t \\
& =(w(T)-u(T), \psi)-\int_{0}^{T}(w(t)-u(t), \nabla \theta(t)) d t=((w(T)-u(T), \psi) .
\end{aligned}
$$

Since $\psi \in C_{0, \sigma}^{\infty}(\Omega)$ is chosen arbitrarily and $C_{0, \sigma}^{\infty}(\Omega)$ is dense in $J_{q^{\prime}}(\Omega)$, we have $w(x, T)=$ $u(x, T)$ for almost every $x \in \Omega$. Since $T>0$ is also chosen arbitrarily, we have $w(x, t)=$ $u(x, t)$ for any $t>0$ in $J_{q}(\Omega)$. In particular, by (23) and (24) we have

$$
\left\|u_{t}(t)\right\|_{L_{q}(\Omega)} \leq C_{\gamma} e^{\gamma t}\|f\|_{W_{q}^{2}(\Omega)}, t>0 .
$$

Applying the Farwig theorem in [5] and cut-off technique to (19), we have

$$
\left\|\nabla^{2} u(t)\right\|_{L_{q}(\Omega)}+\|\nabla \pi(t)\|_{L_{q}(\Omega)} \leq C\left\|u_{t}(t)\right\|_{L_{q}(\Omega)} \leq C_{\gamma} e^{\gamma t}\|f\|_{W_{q}^{2}(\Omega)},
$$

which completes the proof of Theorem 8 .

In order to show Theorem 9, we start with the analysis of the whole space problem:

$$
\begin{aligned}
& u_{t}+L_{k, a} u+\nabla \pi=0 \quad \text { in } \quad \mathbf{R}^{3}, \quad t>0, \\
& \nabla \cdot u=0 \quad \text { in } \mathbf{R}^{3}, \quad t>0, \\
& \left.u\right|_{t=0}=f \quad \text { in } \mathbf{R}^{3},
\end{aligned}
$$

with initial data $f \in J_{q}\left(\mathbf{R}^{3}\right)=\left\{f \in L_{q}\left(\mathbf{R}^{3}\right) \mid \operatorname{div} f=0\right.$ in $\left.\mathbf{R}^{3}\right\}$. We know that the solution $u$ is given by the following formula:

$$
\begin{aligned}
u(t) & =S_{\mathbf{R}^{3}}(t) f=\int_{\mathbf{R}^{3}}(4 \pi t)^{-3 / 2} \exp \left(-\frac{\left|\mathcal{O}(a t) x-y-k \mathbf{e}_{3} t\right|^{2}}{4 t}\right) \mathcal{O}(a t)^{T} P_{\mathbf{R}^{3}} f(y) d y \\
& =\mathcal{F}^{-1}\left[e^{-\left(|\xi|^{2}+i k \xi_{3}\right) t} \mathcal{O}(a t)^{T} \mathcal{F}\left[P_{\mathbf{R}^{3}} f\right](\mathcal{O}(a t) \xi)\right](x) .
\end{aligned}
$$

Here, $P_{\mathbf{R}^{3}}$ is defined as follows:

$$
P_{\mathbf{R}^{3}} f=\mathcal{F}^{-1}\left[\left(\delta_{j k}-\frac{\xi_{j} \xi_{k}}{|\xi|^{2}}\right) \hat{f}(\xi)\right](x)
$$

and $c(f)$ is a constant such that $\int_{\Omega_{R}} Q_{\mathbf{R}^{3}} f d x=0$. Set 


$$
\begin{aligned}
\mathcal{A}_{\mathbf{R}^{3}, a, k}(\lambda) f & =\mathcal{A}(\lambda) f=\int_{0}^{\infty} e^{-\lambda t} S_{\mathbf{R}^{3}}(t) P_{\mathbf{R}^{3}} f d t \\
& =\mathcal{F}^{-1}\left[\int_{0}^{\infty} e^{-\left(\lambda+|\xi|^{2}+i \xi_{3} k\right) t} \mathcal{O}(a t)^{T} \widehat{P_{\mathbf{R}^{3}}} f(\mathcal{O}(a t) \xi) d t\right](x) .
\end{aligned}
$$

The operator $\mathcal{A}_{\mathbf{R}^{3}, a, k}(\lambda)$ is the solution operator to the resolvent problem:

$$
\begin{aligned}
\lambda u+L_{k, a} u+\nabla \pi & =0 \quad \text { in } \quad \mathbf{R}^{3}, \\
\nabla \cdot u & =0 \quad \text { in } \quad \mathbf{R}^{3},
\end{aligned}
$$

which is the resolvent problem of the corresponding non-stationary problem (31). By analyzing the concrete representation formula of $\mathcal{A}_{\mathbf{R}^{3}, a, k}(\lambda)$ we have the following theorem:

Theorem 10. Let $1<q<\infty, k_{0}>0$ and $a_{0}>0$. Assume that $|k| \leq k_{0}$ and $|a| \leq a_{0}$. Let $\gamma>0,0<\epsilon<\pi / 2$ and $N \in \mathbf{N}$ with $N \geq 4$. Set

$$
\begin{aligned}
& \mathbf{C}_{\gamma}=\{\lambda \in \mathbf{C} \mid \operatorname{Re} \lambda \geq \gamma\}, \mathbf{C}_{+}=\{\lambda \in \mathbf{C} \mid \operatorname{Re} \lambda>0\}, \\
& \Sigma_{\epsilon}=\{\lambda \in \mathbf{C} \backslash\{0\}|| \arg \lambda \mid \leq \pi-\epsilon\}, \\
& L_{q, R+2}\left(\mathbf{R}^{3}\right)=\left\{f \in L_{q}\left(\mathbf{R}^{3}\right)^{3} \mid f(x)=0 \text { for } x \notin B_{R+2}\right\}, \\
& \mathcal{L}_{R}\left(\mathbf{R}^{3}\right)=\mathcal{L}\left(L_{q, R+2}\left(\mathbf{R}^{3}\right), W_{q}^{2}\left(\mathbf{R}^{3}\right)^{3}\right) .
\end{aligned}
$$

Then, $R(\lambda) P_{\mathbf{R}^{3}} \in \operatorname{Anal}\left(\mathbf{C}_{+}, \mathcal{L}_{R}\left(\mathbf{R}^{3}\right)\right)$ and there exist three operators:

$$
\mathcal{A}_{1, a}^{N}(\lambda), \tilde{\mathcal{A}}_{1, a}^{N}(\lambda) \in \operatorname{Anal}\left(\mathbf{C} \backslash(-\infty, 0], \mathcal{L}_{R}\left(\mathbf{R}^{3}\right)\right), \quad \mathcal{A}_{2, a}^{N}(\lambda) \in \operatorname{Anal}\left(\mathbf{C}_{+}, \mathcal{L}_{R}\left(\mathbf{R}^{3}\right)\right)
$$

such that

$$
\begin{aligned}
& \mathcal{A}_{\mathbf{R}^{3}, a, k}(\lambda) f=\mathcal{A}_{1, a}^{N}(\lambda)+\mathcal{A}_{2, a}^{N}(\lambda), \\
& \mathcal{A}_{1, a}^{N}(\lambda)=\left(\lambda-\Delta_{\mathbf{R}^{3}}+k \partial_{3}\right)^{-1} P_{\mathbf{R}^{3}}+\tilde{\mathcal{A}}_{1, a}^{N}(\lambda), \\
& \left\|\partial_{x}^{\beta} \mathcal{A}_{1, a}^{N}(\lambda) f\right\|_{L_{q}\left(\mathbf{R}^{3}\right)} \leq C|\lambda|^{-(1-(|\beta| / 2))}\|f\|_{L_{q}\left(\mathbf{R}^{3}\right)}, \\
& \left\|\partial_{x}^{\beta} \tilde{\mathcal{A}}_{1, a}^{N}(\lambda) f\right\|_{L_{q}\left(\mathbf{R}^{3}\right)} \leq C|\lambda|^{-((3 / 2)-(|\beta| / 2))}\|f\|_{L_{q}\left(\mathbf{R}^{3}\right)},
\end{aligned}
$$

for any $\lambda \in \Sigma_{\epsilon}$ with $|\lambda| \geq c_{\epsilon}>0$ ( $c_{\epsilon}$ being some constant depending on $\epsilon$ ) and $\beta$ with $|\beta| \leq 2$, and

$$
\left\|\partial_{x}^{\beta} \mathcal{A}_{2, a}^{N}(\lambda) f\right\|_{L_{q}\left(\mathbf{R}^{3}\right)} \leq C \gamma^{-1}|\lambda|^{-(N / 2)+(|\beta| / 2)}\|f\|_{L_{q}\left(\mathbf{R}^{3}\right)}
$$

for any $\lambda \in \mathbf{C}_{\gamma}$ with $|\lambda| \geq 1$ and $\beta$ with $|\beta| \leq 2$ provided that $f \in L_{q, R+2}\left(\mathbf{R}^{3}\right)$. Here, the constant $C$ depends on $R, a_{0}$ and $k_{0}$ but is independent of a and $k$, and we have set

$$
\left(\lambda-\Delta_{\mathbf{R}^{3}}+k \partial_{3}\right)^{-1} g=\mathcal{F}_{\xi}^{-1}\left[\left(\lambda+|\xi|^{2}+i k \xi_{3}\right)^{-1} \hat{g}(\xi)\right](x) .
$$

Employing a similar argument to that in Hishida and Shibata [24, Section 5], by using Theorem 10 and the representation formula of the resolvent set in $\Omega$, we can show Theorem 9. This is one of our core arguments and we will publish the detailed proof elsewhere.

Now, we shall show Theorem 5 by using Theorem 8 .

Step 1. Given $f \in \mathcal{D}_{q}(\Omega)$, let $\tilde{f} \in \mathcal{D}_{q}\left(\mathbf{R}^{3}\right)=\left\{\tilde{f} \in W_{q}^{2}\left(\mathbf{R}^{3}\right) \mid(\omega \times x) \cdot \nabla \tilde{f} \in L_{q}\left(\mathbf{R}^{3}\right)\right\}$ be an extension of $f$ to the whole space such that $\tilde{f}=f$ on $\Omega$ and $\|\tilde{f}\|_{\mathcal{D}_{q}\left(\mathbf{R}^{3}\right)} \leq C_{q}\|f\|_{\mathcal{D}_{q}(\Omega)}$ where we have set

$$
\|f\|_{\mathcal{D}_{q}(D)}=\|f\|_{W_{q}^{2}(D)}+\|(\omega \times x) \cdot \nabla f\|_{L_{q}(D)}
$$


Let $\varphi \in C_{0}^{\infty}\left(\mathbf{R}^{3}\right)$ such that $\varphi(x)=1$ for $|x| \leq R-2$ and $\varphi(x)=0$ for $|x| \geq R-1$ and set

$$
v(t)=(1-\varphi) S_{\mathbf{R}^{3}}(t) \tilde{f}+\mathbf{B}\left[(\nabla \varphi) \cdot S_{\mathbf{R}^{3}}(t) \tilde{f}\right]
$$

where $\mathbf{B}$ denotes the Bogovski operator satisfying the estimates:

$$
\begin{aligned}
\|\mathbf{B}[(\nabla \varphi) \cdot v]\|_{W_{q}^{j}\left(\mathbf{R}^{3}\right)} & \leq C\|v\|_{W_{q}^{j-1}(\operatorname{supp}(\nabla \varphi))}, \quad j=1,2, \\
\|\mathbf{B}[(\nabla \varphi) \cdot \nabla v]\|_{W_{q}^{j}\left(\mathbf{R}^{3}\right)} & \leq C\|v\|_{W_{q}^{j}(\operatorname{supp}(\nabla \varphi))}, \quad j=0,1,2,
\end{aligned}
$$

(cf. [38], [39], [42], [16]).

Step 2. To obtain the solution $u(t)$ of $(19)$, we set $u(t)=v(t)+w(t)$, and then $w(t)$ and $\pi(t)$ satisfy the equations:

$$
\begin{aligned}
& w_{t}+L_{k, a} w+\nabla \pi=F, \quad \operatorname{div} w=0 \quad \text { in } \Omega \times(0, \infty), \\
& \left.w\right|_{\partial \Omega}=0,\left.\quad w\right|_{t=0}=\varphi f-\mathbf{B}[(\nabla \varphi) \cdot f]=g .
\end{aligned}
$$

Here

$$
\begin{aligned}
F= & -2(\nabla \varphi) \cdot \nabla S_{\mathbf{R}^{3}}(t) \tilde{f}-(\Delta \varphi) S_{\mathbf{R}^{3}}(t) \tilde{f}+k\left(\partial_{3} \varphi\right) S_{\mathbf{R}^{3}}(t) \tilde{f} \\
& -((\omega \times x) \cdot \nabla \varphi) S_{\mathbf{R}^{3}}(t) \tilde{f}+\left(\partial_{t}+L_{k, a}\right) \mathbf{B}\left[(\nabla \varphi) \cdot S_{\mathbf{R}^{3}}(t) \tilde{f}\right] .
\end{aligned}
$$

Observe that

$$
\begin{aligned}
\partial_{t} \mathbf{B}\left[(\nabla \varphi) \cdot S_{\mathbf{R}^{3}}(t) \tilde{f}\right]=\mathbf{B}\left[(\nabla \varphi) \cdot \partial_{t} S_{\mathbf{R}^{3}}(t) \tilde{f}\right] \\
\quad=\mathbf{B}\left[(\nabla \varphi) \cdot \Delta S_{\mathbf{R}^{3}}(t) \tilde{f}\right]+\mathbf{B}\left[(\nabla \varphi) \cdot\left(-k \partial_{3}+((\omega \times x) \cdot \nabla-\omega \times) S_{\mathbf{R}^{3}}(t) \tilde{f}\right)\right] .
\end{aligned}
$$

Therefore, we have

$$
\|F(t)\|_{W_{q}^{2}(\Omega)} \leq C_{\gamma} t^{-1 / 2} e^{\gamma t}\|f\|_{\mathcal{D}_{q}(\Omega)}, \quad\|F(t)\|_{L_{q}(\Omega)} \leq C_{\gamma} t^{-1 / 2} e^{\gamma t}\|f\|_{L_{q}(\Omega)} .
$$

If we write

$$
w(t)=S_{\Omega}(t) g+\int_{0}^{t} S_{\Omega}(t-s) F(s) d s
$$

then by Theorem 14 we have

$$
\begin{gathered}
w(t) \in C^{0}\left([0, \infty), W_{q}^{2}(\Omega)\right) \cap C^{1}\left([0, \infty), L_{q}(\Omega)\right) \\
\|w(t)\|_{W_{q}^{2}(\Omega)}+\left\|w_{t}(t)\right\|_{L_{q}(\Omega)} \leq C_{\gamma} e^{\gamma t}\|f\|_{\mathcal{D}_{q}(\Omega)}, \quad\|w(t)\|_{L_{q}(\Omega)} \leq C_{\gamma} e^{\gamma t}\|f\|_{L_{q}(\Omega)} .
\end{gathered}
$$

Therefore, we can construct a solution

$$
u(t) \in C^{0}\left([0, \infty), W_{q}^{2}(\Omega)\right) \cap C^{1}\left([0, \infty), L_{q}(\Omega)\right)
$$

which satisfies the estimate:

$$
\|u(t)\|_{W_{q}^{2}(\Omega)}+\left\|u_{t}(t)\right\|_{L_{q}(\Omega)} \leq C_{\gamma} e^{\gamma t}\|f\|_{\mathcal{D}_{q}(\Omega)}, \quad\|u(t)\|_{L_{q}(\Omega)} \leq C_{\gamma} e^{\gamma t}\|f\|_{L_{q}(\Omega)} .
$$

If we define $\left\{T_{k, a}(t)\right\}_{t \geq 0}$ by the formula: $T(t) f=u(t)$, then the uniqueness of solutions and the denseness of $\mathcal{D}_{q}(\Omega)$ in $J_{q}(\Omega)$ imply that $\left\{T_{k, a}(t)\right\}_{t \geq 0}$ is a $C^{0}$ semigroup on $J_{q}(\Omega)$. Since we can show that the resolvent set of $\mathcal{L}_{q}$ contains the complex plane with positive real part, the generator of $\left\{T_{k, a}(t)\right\}_{t \geq 0}$ is $\mathcal{L}_{q}$, which completes the proof of Theorem 5 .

5. An idea of a proof of Theorem 6. In order to prove Theorem 6, the main step is to show the following local energy decay estimate. 
Theorem 11 (Local Energy Decay). Let $1<q<\infty$. Then, we have

$$
\left\|\partial_{t}^{j} T_{k, a}(t) P f\right\|_{W_{q}^{2}\left(\Omega_{R}\right)} \leq C t^{-\frac{3}{2}}\|f\|_{L_{q}(\Omega)}, \quad t>1
$$

for any $f \in L_{q, R-1}(\Omega)$ and $j=0,1$.

Employing a similar argument to that in [24, Section 6], we can show Theorem 11 by using some representation formula of solutions to the resolvent problem in $\Omega$ and the following results for the whole-space resolvent operator $\mathcal{A}_{\mathbf{R}^{3}, a, k}(\lambda)$.

Theorem 12. Let $1<q<\infty, k_{0}>0, a_{0}>0, \gamma_{0}$ and $K \geq 10 a_{0}+2$. Assume that $|k| \leq k_{0},|a| \leq a_{0}$ and $0 \leq \gamma \leq \gamma_{0}$. Set

$$
\begin{aligned}
& \mathcal{L}_{R, \operatorname{comp}}\left(\mathbf{R}^{3}\right)=\mathcal{L}\left(L_{q, R+2}\left(\mathbf{R}^{3}\right), W_{q}^{2}\left(B_{R+3}\right)^{3}\right), \quad[\cdot]_{\mathbf{R}^{3}, R}=\|\cdot\|_{\mathcal{L}_{\left(L_{q, R+2}\left(\mathbf{R}^{3}\right), W_{q}^{2}\left(B_{R+3}\right)^{3}\right)},} \\
& \overline{\mathbf{C}_{+}}=\{\lambda \in \mathbf{C} \mid \operatorname{Re} \lambda \geq 0\} .
\end{aligned}
$$

Then, $\mathcal{A}(\lambda)=\mathcal{A}_{\mathbf{R}^{3}, a, k}(\lambda) \in C\left(\overline{\mathbf{C}_{+}}, \mathcal{L}_{R, \text { comp }}\left(\mathbf{R}^{3}\right)\right)$ and possesses the following properties:

$$
\begin{aligned}
& \sup _{|s| \leq K}[\mathcal{A}(\gamma+i s)]_{\mathbf{R}^{3}, R} \leq C_{\gamma_{0}, a_{0}, K} \\
& \int_{-K}^{K}\left[\left(\partial_{\lambda} \mathcal{A}\right)(\gamma+i s)\right]_{\mathbf{R}^{3}, R}^{p} d s \leq C_{\gamma_{0}, a_{0}, K} \quad(1 \leq p<2) \\
& \sup _{0<|h| \leq 1}|h|^{-1 / 2}\left(\int_{-K}^{K}[\mathcal{A}(\gamma+i(s+h))-\mathcal{A}(\gamma+i s)]_{\mathbf{R}^{3}, R}^{p} d s\right)^{1 / p} \leq C_{\gamma_{0}, a_{0}, K} \\
& \sup _{0<|h| \leq 1}|h|^{-1 / 2} \int_{-K}^{K}\left[\left(\partial_{\lambda} \mathcal{A}\right)(\gamma+i(s+h))-\left(\partial_{\lambda} \mathcal{A}\right)(\gamma+i s)\right]_{\mathbf{R}^{3}, R} d s \leq C_{\gamma_{0}, a_{0}, K}, \\
& \left\|\left(\partial_{\lambda}^{m} \mathcal{A}_{\mathbf{R}^{3}, a}\right)(\gamma+i s)\right\|_{\mathcal{L}\left(L_{q, R+2}\left(\mathbf{R}^{3}\right), W_{q}^{j}\left(B_{R+3}\right)^{3}\right)} \leq C_{\gamma_{0}, a_{0}, K}|s|^{-m-(1-(j / 2))}
\end{aligned}
$$

for $m=0,1,2,3, j=0,1,2$ and $s \in \mathbf{R}$ with $|s| \geq K-2$, and

$$
\begin{aligned}
& \lim _{\gamma \rightarrow 0+} \sup _{s \in \mathbf{R}}[\mathcal{A}(\gamma+i s)-\mathcal{A}(i s)]_{\mathbf{R}^{3}, R}=0 \\
& \lim _{\gamma \rightarrow 0+} \int_{-\infty}^{\infty}\left[\left(\partial_{\lambda} \mathcal{A}\right)(\gamma+i s)-\left(\partial_{\lambda} \mathcal{A}\right)(i s)\right]_{\mathbf{R}^{3}, R}=0 \\
& \lim _{R \rightarrow \infty} R^{-1} \int_{R \leq|x| \leq 2 R}|[\mathcal{A}(\lambda) f](x)|^{2} d x=0 \quad(k \neq 0) \\
& \lim _{R \rightarrow \infty} R^{-2} \int_{R \leq|x| \leq 2 R}|[\mathcal{A}(\lambda) f](x)|^{2} d x=0 \quad(k=0) \\
& \left\|\mathcal{A}\left(\lambda_{1}\right) f-\mathcal{A}\left(\lambda_{2}\right) f\right\|_{W_{q}^{2}\left(B_{R+3}\right)} \leq C_{q, R}\left|\lambda_{1}-\lambda_{2}\right|^{1 / 4}\|f\|_{L_{q}\left(\mathbf{R}^{3}\right)}
\end{aligned}
$$

for any $\lambda, \lambda_{1}$ and $\lambda_{2} \in \overline{\mathbf{C}_{+}}$provided that $f \in L_{q, R+2}\left(\mathbf{R}^{3}\right)$.

Since by Young's inequality we see easily that

$$
\left\|\nabla^{j} S_{\mathbf{R}^{3}}(t) f\right\|_{L_{r}\left(\mathbf{R}^{3}\right)} \leq C_{q, r} t^{-\frac{j}{2}-\frac{3}{2}\left(\frac{1}{q}-\frac{1}{r}\right)}\|f\|_{L_{q}\left(\mathbf{R}^{3}\right)}
$$

for $1<q \leq r \leq \infty$ with $q \neq \infty$ and $t>0$, employing the same argument as in [24, section 7], combining (33) with Theorem 11 and Theorem 9 by cut-off technique, we have Theorem 6 . We will show the detailed proof of Theorem 6 elsewhere. 


\section{References}

[1] W. Borchers, Zur Stabilität und Faktorisierungsmethode für die Navier-Stokes Gleichungen inkompressibler viskoser Flüssigkeiten, Habilitationsschrift, Universität Paderborn, 1992.

[2] Z. Chen and T. Miyakawa, Decay properties of weak solutions to a perturbed NavierStokes system in $\mathbf{R}^{n}$, Adv. Math. Sci. Appl. 7 (1997), 741-770.

[3] P. Cumsille and M. Tucsnak, Strong solutions for the Navier-Stokes flow in the exterior of a rotating obstacle, preprint.

[4] E. Dintelmann, M. Geissert and M. Hieber, Strong $L^{p}$ solutions to the Navier-Stokes flow past moving obstacles: the case of several obstacles and time dependent velocity, TU Darmstadt, Fachbereich Mathematik, preprint, 2006.

[5] R. Farwig, An $L^{q}$-analysis of viscous fluid flow past a rotating obstacle, Tôhoku Math. J. 58 (2006), 129-147.

[6] R. Farwig, M. Krbec and Š. Nečasová, A weighted $L^{q}$-approach to Stokes flow around a rotating body, Ann. Univ. Ferrara Sez. VI Sci. Mat. 54 (2008), 61-84.

[7] R. Farwig and T. Hishida, Stationary Navier-Stokes flow around a rotating obstacle, Funkcial Ekvac. 50 (2007), 371-403.

[8] R. Farwig, T. Hishida and D. Müller, $L^{q}$-theory of a singular "winding" integral operator arising from fluid dynamics, Pacific J. Math. 215 (2004), 297-312.

[9] R. Farwig, Š. Nečasová and J. Neustupa, On the essential spectrum of a Stokes-type operator arising from flow around a rotationg body in the $L^{q}$-framework, RIMS Kokyuroku Bessatsu B1 (2007), 93-105

[10] R. Farwig and J. Neustupa, On the spectrum of a Stokes-type operator arising from flow around a rotating body, Manuscripta Math. 122 (2007), 419-437.

[11] G. P. Galdi, On the motion of a rigid body in a viscous liquid: a mathematical analysis with applications, in: Handbook of Mathematical Fluid Dynamics, Volume I, S. Friedlander and D. Serre (eds.), North-Holland, Amsterdam, 2002, 653-791.

[12] G. P. Galdi, Steady flow of a Navier-Stokes fluid around a rotating obstacle, J. Elasticity 71 (2003), 1-31.

[13] G. P. Galdi and A. L. Silvestre, Strong solutions to the Navier-Stokes equations around a rotating obstacle, Arch. Rational Mech. Anal. 176 (2005), 331-350.

[14] G. P. Galdi and A. L. Silvestre, On the steady motion of a Navier-Stokes liquid around a rigid body, Arch. Rational Mech. Anal. 184 (2007), 371-400.

[15] G. P. Galdi and A. L. Silvestre, Existence of time-periodic solutions to the Navier-Stokes equations around a moving body, Pacific J. Math. 223 (2006), 251-267.

[16] M. Geissert, H. Heck and M. Hieber, $L^{p}$-theory of the Navier-Stokes flow in the exterior of a moving or rotating obstacle, J. Reine Angew. Math. 596 (2006), 45-62.

[17] R. B. Gunther, R. T. Hudspeth and E. A. Thomann, Hydrodynamic flows on submerged rigid bodies - steady flow, J. Math. Fluid Mech. 4 (2002), 187-202.

[18] M. Hieber and O. Sawada The Navier-Stokes equations in $\mathbf{R}^{n}$ with linearly growing initial data, Arch. Rational Mech. Anal. 175 (2005), 269-285.

[19] T. Hishida, An existence theorem for the Navier-Stokes flow in the exterior of a rotating obstacle, Arch. Rational Mech. Anal. 150 (1999), 307-348.

[20] T. Hishida, The Stokes operator with rotation effect in exterior domains, Analysis 19 (1999), 51-67. 
[21] T. Hishida, $L^{2}$ theory for the operator $\Delta+(k \times x) \cdot \nabla$ in exterior domains, Nihonkai Math. J. 11 (2000), 103-135.

[22] T. Hishida, The nonstationary Stokes and Navier-Stokes flows through an aperture, in: Contributions to Current Challenges in Mathematical Fluid Mechanics, Adv. Math. Fluid Mech. 3, Birkhäuser, Basel, 2004, 79-123.

[23] T. Hishida, $L^{q}$ estimates of weak solutions to the stationary Stokes equations around a rotating body, J. Math. Soc. Japan 58 (2006), 743-767.

[24] T. Hishida and Y. Shibata, $L_{p}-L_{q}$ estimate of the Stokes operator and Navier-Stokes flows in the exterior of a rotating obstacle, To appear in Arch. Rational Mech. Anal.

[25] G. Kirchhoff, Über die Bewegung eines Rotationskörpers in einer Flüssigkeit, Crelle J. 71 (1869), 237-281.

[26] S. Kračmar, Š. Nečasová and P. Penel, Estimates of weak solutions in anisotropically weighted Sobolev spaces to the stationary rotating Oseen equations, IASME Transactions 6 (2005), 854-861.

[27] S. Kračmar, Š. Nečasová and P. Penel, On the weak solution to the Oseen-type problem arising from flow aroung a rotating rigid body in the whole space, WSEAS Transactions of Mathematics 3 (2006), 243-251.

[28] Š. Nečasová, On the problem of the Stokes flow and Oseen flow in $\mathbf{R}^{3}$ with Coriolis force arising from fluid dynamics, IASME Transactions 2 (2005), 1262-1270.

[29] Š. Nečasová, Asymptotic properties of the steady fall of a body in viscous fluids, Math. Meth. Appl. Sci. 27 (2004), 1969-1995.

[30] J. A. San Martín, V. Starovoitov and M. Tucsnak, Global weak solutions for the twodimensional motion of several rigid bodies in an incompressible viscous fluid, Arch. Rational Mech. Anal. 161 (2002), 113-147.

[31] D. Serre, Chute libre d'un solide dans un fluide visqueux incompressible. Existence, Japan J. Appl. Math. 4 (1987), 99-110.

[32] A. L. Silvestre, On the existence of steady flows of a Navier-Stokes liquid around a moving rigid body, Math. Meth. Appl. Sci. 27 (2004), 1399-1409.

[33] T. Takahashi and M. Tucsnak, Global strong solutions for the two-dimensional motion of an infinite cylinder in a viscous fluid, J. Math. Fluid Mech. 6 (2004), 53-77.

[34] E. A. Thomann and R. B. Guenther, The fundamental solution of the linearized NavierStokes equations for spinning bodies in three spatial dimensions-time dependent case, J. Math. Fluid Mech. 8 (2006), 77-98.

[35] W. Thomson (Lord Kelvin), Mathematical and Physical Papers, Vol 4, Cambridge University Press 1982.

[36] H. F. Weinberger, On the steady fall of a body in a Navier-Stokes fluid, in: Proc. Symp. Pure Mathematics 23 (1973), 421-440.

[37] H. F. Weinberger, Variational properties of steady fall in Stokes flow, J. Fluid Mech. 52 (1972), 321-344.

[38] M. E. Bogovskiı, Solution of the first boundary value problem for the equation of continuity of an incompressible medium, Soviet Math. Dokl. 20 (1979), 1094-1098.

[39] M. E. Bogovskiı̌, Solution of some vector analysis problems connected with operators div and grad, Trudy Seminara S. L. Soboleva, 1, Akademia Nauk SSR, Sibirskoe Otdelenie Matematiki, Nowosibirsk, 1980, 5-40 (in Russian).

[40] D. Fujiwara and H. Morimoto, An $L_{r}$-theory of the Helmholtz decomposition of vector fields, J. Fac. Sc. Univ. Tokyo, Sect. Math. 24 (1977), 685-700. 
[41] M. Geissert, H. Heck and M. Hieber, On the equation div $u=g$ and Bogovskiu's operator in Sobolev spaces of negative order, in: Operator Theory: Advances and Applications 169 (2006), 113-121.

[42] G. P. Galdi, An Introduction to the Mathematical Theory of the Navier-Stokes equations, Vol. I: Linear Steady Problems, Vol. II: Nonlinear Steady Problems. Springer Tracts in Nat. Ph. 38, 39, Springer, New York, 1994; 2nd edition, 1998.

[43] H. Iwashita, $L_{q}-L_{r}$ estimates for solutions of the nonstationary Stokes equations in an exterior domain and the Navier-Stokes initial value problem in $L_{q}$ spaces, Math. Ann. 285 (1989), 265-288.

[44] T. Kato, Strong $L^{p}$ - solutions of the Navier-Stokes equation in $\mathbf{R}^{m}$ with applications to weak solutions, Math. Z. 187 (1984), 471-480.

[45] T. Kobayashi and Y. Shibata, On the Oseen equation in the three dimensional exterior domains, Math. Ann. 310 (1998), 1-45.

[46] P. Maremonti and V. A. Solonnikov, On nonstationary Stokes problem in exterior domains, Ann. Sc. Norm. Sup. Pisa, 24 (1997), 395-449.

[47] T. Miyakawa, On non-stationary solutions of the Navier-Stokes equations in an exterior domain, Hiroshima Math. J. 12 (1982), 115-140.

[48] Y. Shibata, On an exterior initial boundary value problem for Navier-Stokes equations, Quart. Appl. Math. 57 (1999), 117-155.

[49] Y. Shibata, Time-global solutions of nonlinear evolution equations and their stability, Amer. Math. Soc. Transl. (2) 211 (2003), 87-105.

[50] C. G. Simader and H. Sohr, The Helmholtz decomposition in $L^{q}$ and related topics, in: Mathematical Problems Related to the Navier-Stokes Equation, G. P. Galdi (ed.), Advances in Maths. Appl. Sci. 11, World Scientific, 1992, 1-35. 
